effects on human persnal Journal on Man-

ence, 73, 31-68.

imes) worth ten thou-

A review of research. ?.

sally validating funcn (editors). The psy-

$\mathrm{h}$ ten thousand words?

Wesley. graphical representa5, 185-213.

raphical and linguistic 7-140.

nts perceive in their

naps: Layers of Tepre-

1s. In F. T. Marchese lerlag.

iattis (Editor), Spatial

uage. In C. Freksa, C isciplinary approach to 75. Berlin: Springer-

In $\mathbf{H}$. Mandl and J. R

Pp. 125-143. North

f graphic communica-

(1999). In C. Freksa \& D.M.Mark (Eds.), Spatial information theory: Cognitve and computational foundations of geographic information science (pp. 65-82). Lecture Notes in Computer Science 1661.

\title{
Elements of Good Route Directions in Familiar and Unfamiliar Environments
}

\author{
Kristin L. Lovelace ${ }^{1}$, Mary Hegarty ${ }^{1}$ \\ and Daniel R. Montello 2 \\ 1 University of California Dept. of Psychology, Santa Barbara, CA 93106 \\ lovelace@psych.ucsb.edu, hegarty@psych.ucsb.edu \\ 2 University of California, Dept. of Geography , Santa Barbara, CA 93106 \\ montello@geog.ucsb.edu
}

\begin{abstract}
Route directions are instructions, primarily verbal, that explain how to get from one place to another. The current study examines several methods for assessing the quality of verbal route directions by characterizing them in terms of the number of elements (such as landmarks, segments or turns) and by subjective ratings of their goodness. Route directions for routes which were both familiar and unfamiliar to the participant were studied. Subjective ratings of the quality of route directions were reliable and consistent across individuals. More complete route directions were rated as being of higher quality. For both routes, inclusion of more segment and turn mentions were correlated with higher quality route directions. Good route descriptions for familiar versus unfamiliar routes differed in terms of the types of landmarks included.
\end{abstract}

Keywords: Spatial language, Route directions, Familiarity

\section{Introduction}

Route directions are responses to a query of the type 'how can I get from A to B?'. What does it mean to give someone "good" route directions? The issue of how to recognize and/or produce 'good' route directions is of great importance for many practical applications (e.g. navigational information for the disabled, personalized or automated tourist information, in-vehicle navigation systems) as well as of theoretical interest. A characterization of what constitutes high quality route directions, and how this differs with level of environmental familiarity, are the broad goals of the current study.

To date there have been few studies on route directions. Most of the earliest research was by linguists (Klein, 1983; Talmy, 1983; Wunderlich and Reinelt, 1982), but the issue has also been studied by psychologists, computer scientists and geographers (Allen, 1997; Couclelis, 1996; Denis, 1997; Denis, Pazzaglia, 
Cornoldi \& Bertolo,j1999; Golding, Graesser \& Hauselt, 1996; Mark, 1987; Reisbeck, 1980; Streeter, Vitello and Wonsiewicz, 1985; Vanetti and Allen, 1988).

Four models of the route direction production task have been proposed, with very similar components. Wunderlich and Reinelt (1982) proposed a model with four phases: Initiation, Route Description, Securing and Closure. All but the second phase are interactions between the route direction producer and the 'questioner'. Couclelis' (1996) model has five major stages: Initiation, Representation, Transformation, Symbolization, and Termination. The first and last stages are relevant to the interpersonal communication of a request for route directions. The third, fourth and fifth stages involve the translation of a spatial representation into verbal output to solve the given problem.

Allen's (1997) model has four phases': Initiation, Route Description, Securing and Closure. Again the first, third and fourth stages deal mainly with the rituals of the route direction exchange, while the actual production of information occurs in the Route Description phase. Allen's model does not allude to underlying spatial or verbal representations or abilities, but rather focuses on the elements or attributes of the route directions themselves. The fourth model, formulated by Denis (1997; Denis et al., 1999), is composed of three 'cognitive operations': activating relevant spatial knowledge, determining a route through the activated representation, and formulating procedural verbal output. These three steps are very similar to the third, fourth and fifth steps of the Couclelis model.

A simplified theoretical model of route direction production, consistent with the four models described above, consists of 3 major steps (see Figure 1). The first step is activation of a representation, the spatial knowledge of the environment to be described, at the appropriate scale for the route. This knowledge is presumed by most researchers to be stored in a non-linguistic format. The second step requires the choice of a specific route through that environment. This choice process could be based on one or more criteria, such as the mode of travel (e.g. walking or driving), desired route characteristics (e.g. fastest or most scenic), and the expected level of knowledge of the receiver of the route directions. The third step is a translation of the chosen route through the environment into a set of verbal instructions. These three steps are the central part of a discourse which usually occurs between the giver and seeker of route directions. Because we use written directions in the current research, the initiation and termination activities are not relevant, and are not included in the model. We focus on the third step in Figure 1: the verbal output.

To approach the question of what characterizes good route directions, we examine quality of route directions both subjectively, via a rating scale, and quantitatively, via frequencies of route direction elements. We also explore differences in route directions produced about familiar versus unfamiliar environments.

The phase names are identical to Wunderlich and Reinelt's but the content is different. 
96; Mark, 1987; anetti and Allen,

n proposed, with sed a model with ire. All but the oroducer and the ges: Initiation, n. The first and request for route ation of a spatial

sription, Securing $y$ with the rituals iformation occurs ide to underlying 1 the elements or il, formulated by itive operations': ugh the activated e three steps are Iodel.

nsistent with the ure 1). The first z environment to edge is presumed The second step nt. This choice de of travel (e.g. nost scenic), and tions. The third int into a set of discourse which Because we use ination activities the third step in

e directions, we ating scale, and $\mathrm{Ve}$ also explore rsus unfamiliar

intent is different.

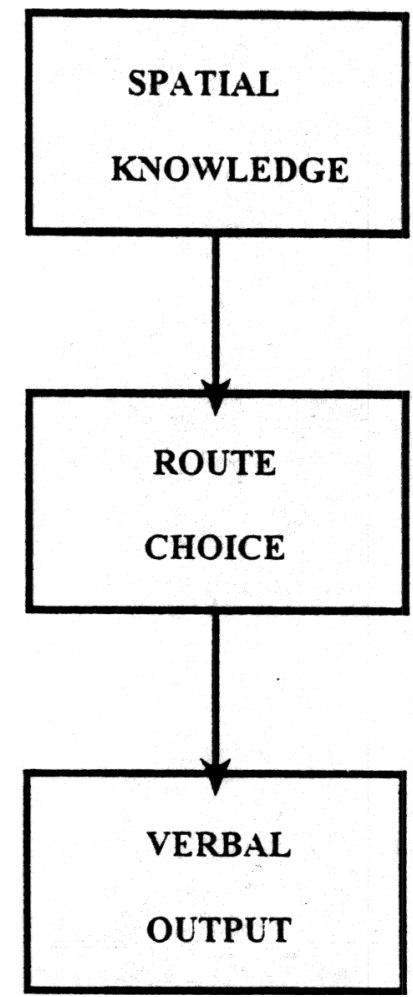

Fig. 1. Simplified model of stages in route direction production

\subsection{Quality of Route Directions}

Quality of route directions can be measured in a number of ways. First, the quality may be a function of the absolute number of various elements included in the verbal output (e.g. landmarks, turns, descriptive information), which are presumed to aid interpretation or route following. Second, good route directions can also be measured subjectively, by asking people to rate the directions with a rating scale. Finally, route direction quality may be measured functionally, in terms of how well the directions facilitate completion of the wayfinding task they were produced for. All of these types of measures have been used in the past, and it is the convergence of the methods which is most useful in determining quality. The current study focuses on the first and second methods.

\subsection{Quantifying quality: Characteristics of route directions}

There are a large number of elements in verbal route directions which may be potentially relevant to their quality. Route directions usually consist of a sequential description of the route, including physical elements and basic motor activities (walk, turn [left], etc.). Kuipers (1978) calls these 'view-action pairs', and considers each view-action pair the most basic building block of route 
knowledge. Similarly, Allen (1997) breaks route direction elements into directives (movement or state-of-being verbs) and descriptives (environmental features: paths, choice points, and landmarks).

The route itself is broken down into segments, verbally represented as one or more phrases (Couclelis, 1996). There is no consensus on what these phrases should include (perhaps due to the variety of environments and research questions asked) (Allen, 1997; Couclelis, 1996; Denis, 1997; Denis et al., 1999; Vanetti and Allen, 1988). The organization of written route directions is most commonly linear -- the transformation of potentially 3-D environmental knowledge into a 2D sequentially experienced route -- although survey-type description is also possible. The correct ordering of segments is their most important organizational feature.

There is also no accepted definition of what constitutes 'good route directions'. Several researchers (Allen, 1997; Denis et al., 1999; Mark, 1987; Mark and Gould, 1995; Streeter et al., 1985; Waller, 1985; Wunderlich and Reinelt, 1982) have made suggestions about important aspects of route direction components, for the most part based on functional criteria. These aspects include a) priming the traveler for upcoming choice points, b) mentioning landmarks at choice points, c) giving "you've gone too far if" statements in case a choice point is missed, d) giving landmarks rather than street names, e) giving distances between choice points, $f$ ) telling the traveler which way to proceed at a choice point, $g$ ) providing information to allow recovery from errors, h) providing clearly linear information (e.g. using 'then', and focusing on a sequential rather than global view), and i) providing a limited amount of redundant information. Some of these suggestions are based on empirical evidence, while others are based on thought experiments, intuition or are lists of common-sense ideas which have not been tested. While we believe that intuition can be a very powerful tool for subjectively recognizing route direction quality, we also wish to examine the empirical relationship between route direction quality and objective measures of route direction elements.

\subsection{Spatial Knowledge: Familiar and Unfamiliar environments}

The first stage of the simple model of route directions (Figure 1) is the Spatial Knowledge which an individual brings to bear on the production (or comprehension) of route directions. Familiarity with an area is probably positively correlated with the completeness of this internal spatial representation. The quality and detail of this representation is also crucial to Route Choice, stage 2 of the proposed model.

As familiarity (and therefore spatial knowledge) increases, spatial description tasks (such as production of route directions) should become easier. Unfamiliar areas are likely to have simplified, incomplete, incorrect or even non-existent spatial representations, and should result in route directions of significantly lower completeness, detail and correctness. We tested this hypothesis by asking people to describe routes in both familiar and unfamiliar locations of campus. 
tion elements into ives (environmental

spresented as one or what these phrases $\mathrm{d}$ research questions al., 1999; Vanetti is most commonly nowledge into a 2lescription is also rtant organizational d route directions'. , 1987; Mark and and Reinelt, 1982) on components, for ude a) priming the It choice points, c) oint is missed, d) es between choice soint, g) providing linear information Jbal view), and i) these suggestions ught experiments, een tested. While tively recognizing irical relationship lirection elements.

\section{vironments}

1) is the Spatial production (or area is probably al representation. Ite Choice, stage

satial description sier. Unfamiliar ven non-existent ;nificantly" lower iy asking people ipus.

\section{Main Experiment}

A pilot study using 36 route directions determined that route directions can be reliably scored for quality. Route directions were scored for quality on a 1 (worst) to 5 (best) scale. No explicit definitions or criteria were given for 'quality'; the ratings were based on the rater's subjective impression. A small subset of directions were scored by 5 raters, with $86 \%$ agreement among the raters ${ }^{2}$. All 36 sets were then rated by two raters, with an inter-rater agreement of $75 \%$.

Based on these findings, an experiment was designed to answer several further questions about 'good' route directions. First, what are the characteristics of good route directions? Second, in what ways are route directions for familiar and unfamiliar routes different? To answer these questions, we a) collected route descriptions for both a familiar and unfamiliar route, b) subjectively rated both sets of route directions, and 3) quantitatively measured elements of the route descriptions and correlated the frequency of each element with the route description ratings.
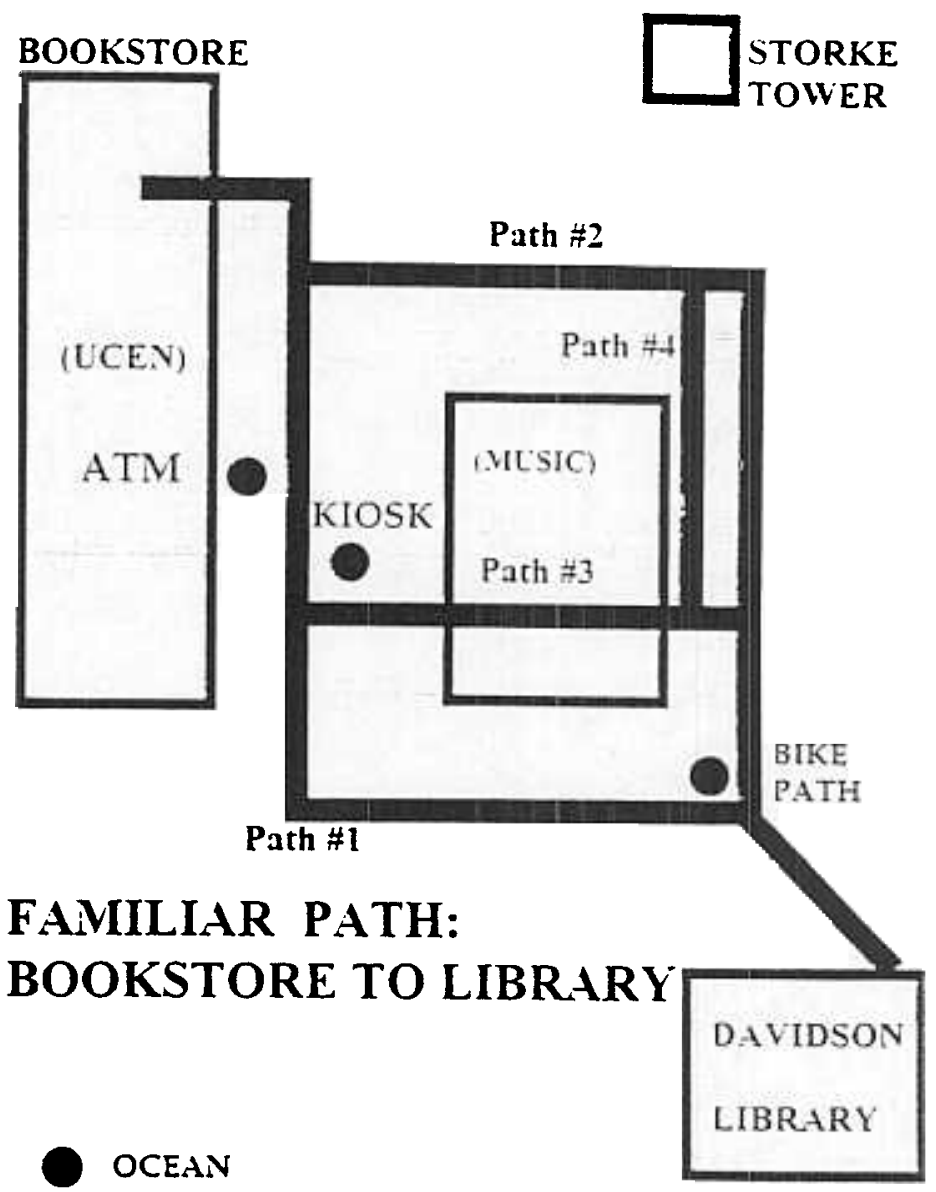

Fig. 2. Familiar Route with Landmark Types. Choice Point: Bike Path. OnRoute (not at Choice Point): ATM. Potential Choice Point: Kiosk. Off Route: Ocean

\footnotetext{
${ }^{2}$ Interrater reliability for the 5 raters is the mean of the IRR for each pair of raters.
} 
Compared to previous studies (Allen, 1996; Denis, 1997; Denis et al., 1999; Vanetti and Allen, 1988) this study includes a more thorough exploration of the kinds and locations of landmarks included in route directions. In the past, landmarks have been defined as 1) 'at a choice point' (at any point where a potential change of direction exists) or 2) non-choice point landmarks. In the current research we will make a distinction between landmarks that are located at potential turning points but are not used on the route being described (called here 'potential choice point' landmarks), and landmarks that are located at choice points which are used on the route described (called here 'choice point' landmarks). Nonchoice point landmarks are similarly broken down into those 'on route' (along the path of travel but not at a potential or used choice point) and those 'off-route' (not contiguous to the path followed but of some orientation value, whether visible or not, such as mountains, ocean, or out of view buildings). Examples of these 4 types are shown in Figure 2.

Three specific claims from previous route direction research will be addressed. Intuitively, it would seem that more complete information is necessarily better. However, Denis et al. (1999) has questioned this, finding instead that route directions rated as best are of moderate length, containing neither too much nor too little information. These conflicting hypotheses will be examined. A second claim is that the only landmarks that are important to route direction quality are those at choice points (re-orientation points on the path, whether used or not) (Denis et al., 1999). A third claim is that the density of landmarks mentioned increases near the end of the route description in order to allow identification of the correct end point of the route (Allen, 1997).

As a pretest, prior to the main experiment, 64 participants rated their familiarity with areas of campus. Two routes were chosen in areas of low familiarity. Average time on campus was 1.3 quarters for those who knew the area of neither route, so participation in the main study was limited to freshmen.

\subsection{Method}

Thirty one freshmen (17 males, 14 females) from an introductory psychology and an introductory human geography course completed two testing sessions for course credit. The mean age of participants was 18.7 years.

In the first session a number of spatial and verbal psychometric tasks were administered and route directions were collected between two familiar campus locations (see schematic in Figure 3a). Only the results for the route direction task will be reported here.

Materials for the second session consisted of blank paper for writing route directions (for unfamiliar route number one, the Description Route), a set of twenty photographs, and an enlarged map of a section of campus (for unfamiliar route number two, the Retrace Route). Two routes in an infrequently used area of the campus were created by the pretest explained above. The Description Route (schematic in Figure 3b) included 14 segments and 13 turns (with 11

unconstrained turn Route included 18

A set of twenty

measure on the $\mathbf{R e}^{1}$

Retrace Route, anc

photographs were page.s

In the first sess written tasks in s experimenter met the courtyard of ai The participants $r$ minutes duration route. Participant task to-test their were answered, p:

At the end of $t$ route just walked They were instru campus very wel turns, and any lar When the part the beginning of and told that at $\mathrm{tl}$ knowledge. Aga the end of the sec photographs, anc photo ahead of $t]$

For the final $\mathbf{t}$ asked to walk fi route they had confused they cr went off the rol them to the rov and noted the fo off the route, 2 points.

Finally, the f The average tin

2.1.1 Route A group of for quality on agreement on $\mathbf{t}$ route direction: 
lenis et al., 1999; exploration of the is. In the past, ly point where a ndmarks. In the that are located at zribed (called here $\mathrm{d}$ at choice points andmarks). Nonroute' (along the se 'off-route' (not hether visible or mples of these 4

vill be addressed. essarily better. istead that route ir too much nor lined. A second :ction quality are er used or not) narks mentioned identification of

ants rated their $\mathrm{n}$ areas of low who knew the $\mathrm{d}$ to freshmen.

rry psychology ig sessions for

ric tasks were miliar campus route direction

writing route ute), a set of for unfarmiliar $y$ used area of 'ription Route ns (with 11 unconstrained turns; that is, turns where $2+$ choices were available). The Retrace Route included 18 segments and 17 turns (with 15 unconstrained turns).

A set of twenty 4 "x 6" color photographs were used for a scene recognition measure on the Retrace Route. The photographs were of 10 locations along the Retrace Route, and 10 photographs of similar locations on the campus. The photographs were mounted in a photograph album, with one photograph per page.

In the first session, lasting about 50 minutes, the participants completed the written tasks in small groups in the laboratory. In the second session, the experimenter met the participants individually at the laboratory, then led them to the courtyard of an adjacent building to the beginning of the first unfamiliar route. The participants were told that they would be guided along a walk of about 8 minutes duration. They were asked to follow behind the experimenter on the route. Participants were told that at the end of the route, they would be given a task to test their spatial knowledge of the route traversed. After any questions were answered, participants followed the experimenter along the route (Figure 3b). - At the end of the route, the participants were asked to write directions for the route just walked, imagining they were starting at the beginning of the route. They were instructed to write the directions for someone who didn't know the campus very well, and to include information about sections of the route, the turns, and any landmark information they thought would be helpful.

When the participants were finished writing they were led a short distance to the beginning of the second novel route. They were given the same instructions, and told that at the end of this route they would be given a different test of route knowledge. Again the participants followed the experimenter along the route. At the end of the second route the participants were asked to look at each of the 20 photographs, and to tell the experimenter if they had seen the view shown in the photo ahead of them on the route they had just walked.

For the final task, participants were asked to retrace the second route: they were asked to walk from the end of the route back to the beginning, using the same route they had just followed. Participants were told that if they felt lost or confused they could ask, and they would be told the correct way to go, and if they went off the route the experimenter would stop them after 10 steps and return them to the route. During the retrace the experimenter followed the participant and noted the following relevant behaviors of the participants: 1) number of steps off the route, 2) number of times off the route, and 3) wrong turns at choice points.

Finally, the participant was debriefed, and any further questions were answered. The average time to complete the second session was 50 minutes.

\subsubsection{Route Direction Scoring}

A group of three naive raters rated all familiar and unfamiliar route directions for quality on a 1-5 scale as in the pilot experiment. These raters had $69 \%$ agreement on the familiar route directions, and $73 \%$ agreement on the unfamiliar route directions. 
Both sets of directions were also coded for the inclusion of ten elements (e.g. landmarks, segments, turns, overall length and errors). Inter-rater reliability of the coding of these variables showed an average $83 \%$ agreement between two trained coders (the first and second authors).

There were four landmark variables: 1) choice point landmarks (landmarks at any turn along the path which was used in this route), 2) potential choice point landmarks ( landmarks at a potential turn along the path which was not used for this route), 3) on route landmarks (landmarks along the route and not at any choice point) and 4) off route landmarks (distant, often non-visible objects such as the ocean, mountain range or student housing area).

There were three choice point variables: 1) the number of turn statements (e.g. those that include the word "turn"), 2) the number of choice points at which landmarks were mentioned, and 3) the number of turn statements for which a descriptor was used (right/left, north/south, or towards an environmental feature).

We also counted the number of segments mentioned (use of terms such as 'walk along, follow the path, keep going', etc.) and the number of errors in the content of the route directions given (such as wrong names for buildings, or telling someone to turn the wrong direction, misplacing a landmark on the route). Omission of information was not considered an error. Finally, the number of words was counted.

\section{Results}

There are several main questions of interest. Most basically, what are the general characteristics of route directions in terms of their elements (e.g. turns, landmarks)? In what ways do the elements of route directions for familiar and unfamiliar routes differ? And which elements are used in highly rated route directions?

\subsection{General Route Direction Characteristics}

Although there are a number of different ways to walk between the two endpoints of the familiar route (see Figure 2), 74\% (23) of the participants chose to describe the route in Figure $3 \mathrm{a}$. This route was used for the following analysis.

Examination of the frequency of mention of specific turns and segments in the two sets of route directions (Figures $3 a \& 3 b$ ) shows that longer segments are more frequently mentioned than shorter ones, and turns towards the end of the route appear to be less frequently mentioned. Some possible reasons for the mention of a turn or segment are addressed in the discussion. Both the familiar and unfamiliar route directions were quite incomplete, shown by the percentage of turns and segments included (see Table 1).

Fig.3b. 
ten elements (e.g. ater reliability of nent between two

rks (landmarks at ntial choice point was not used for $\mathrm{e}$ and not at any le objects such as

1 statements (e.g. points at which lents for which a imental feature). of terms such as ir of errors in the for buildings, or ark on the route). , the number of

lly, what are the lents (e.g. turns, ; for familiar and ghly rated route

retween the two articipants chose llowing analysis. segments in the yer segments are $s$ the end of the reasons for the 1 the familiar and he percentage of

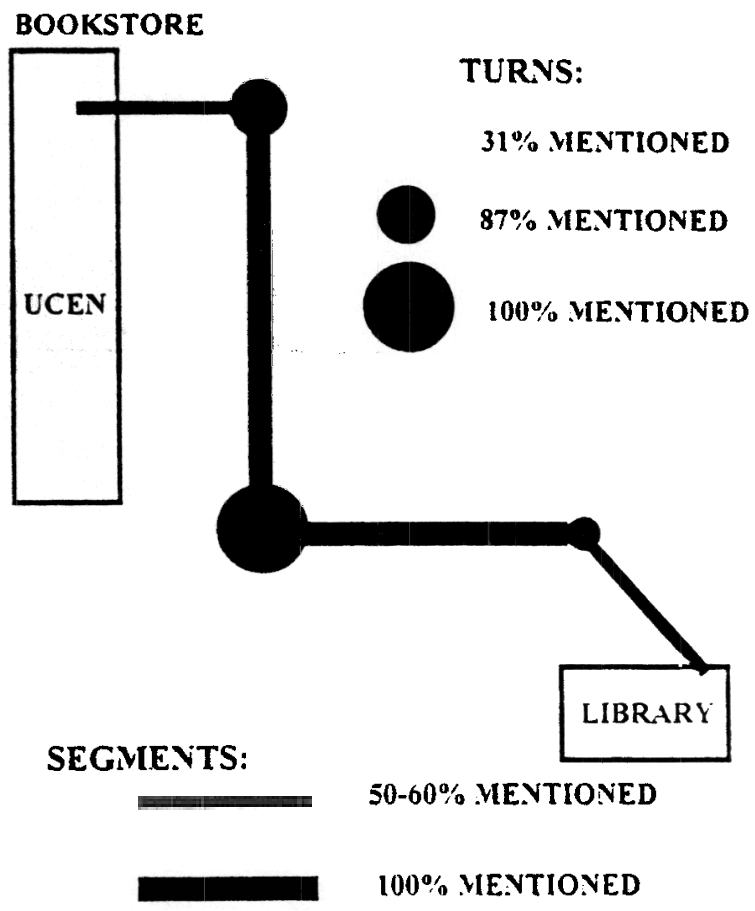

Fig. 3a. Frequency of Segment and Turn Mention, Familiar Route

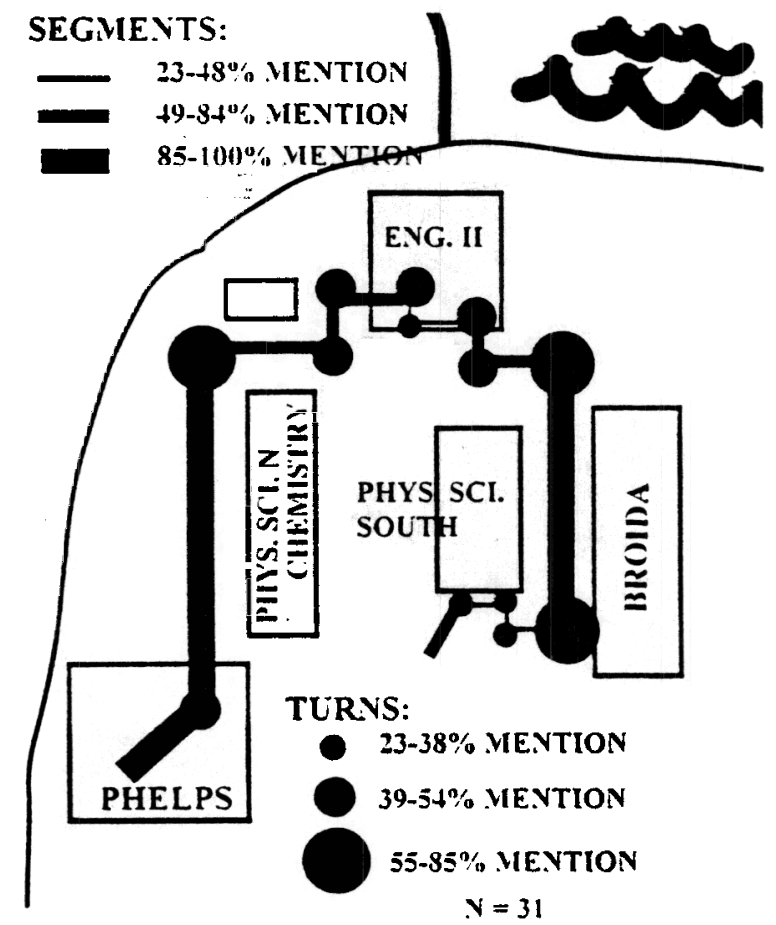

Fig.3b. Frequency of Segment and Turn Mention, Unfamiliar Route 


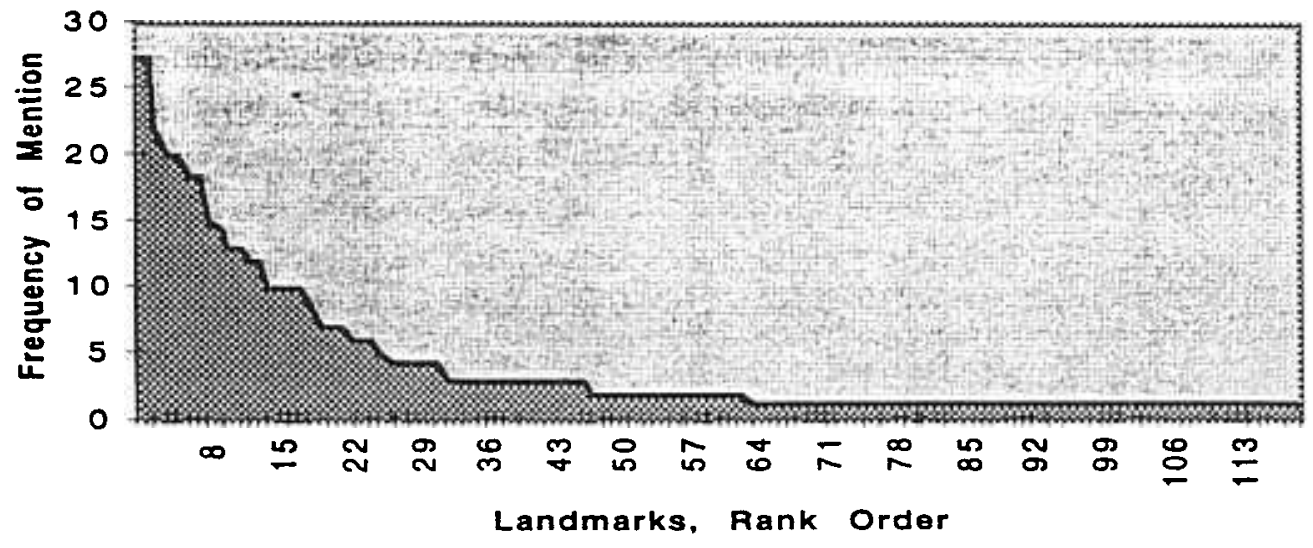

frequently mentios points where there of mentions seen landmark mention the unfamiliarity

\subsection{Characteri}

The elements compared in Tab similar means an route) showing segments, and ty for the other mea

The percentag

There is no easy way to assess the potential number of landmarks along the route. However, 119 different landmarks were mentioned along the unfamiliar route by the 31 participants, with only a small number (16) being mentioned by $30 \%$ or more of the participants. The frequency of mention of all 119 landmarks is given in Figure 4; the frequency of mention decreases steadily, and is relatively flat after the first 25 landmarks.

The distribution of landmark mention for the unfamiliar route along the segments and at the choice points is shown in Figure 5. Landmarks appear to be

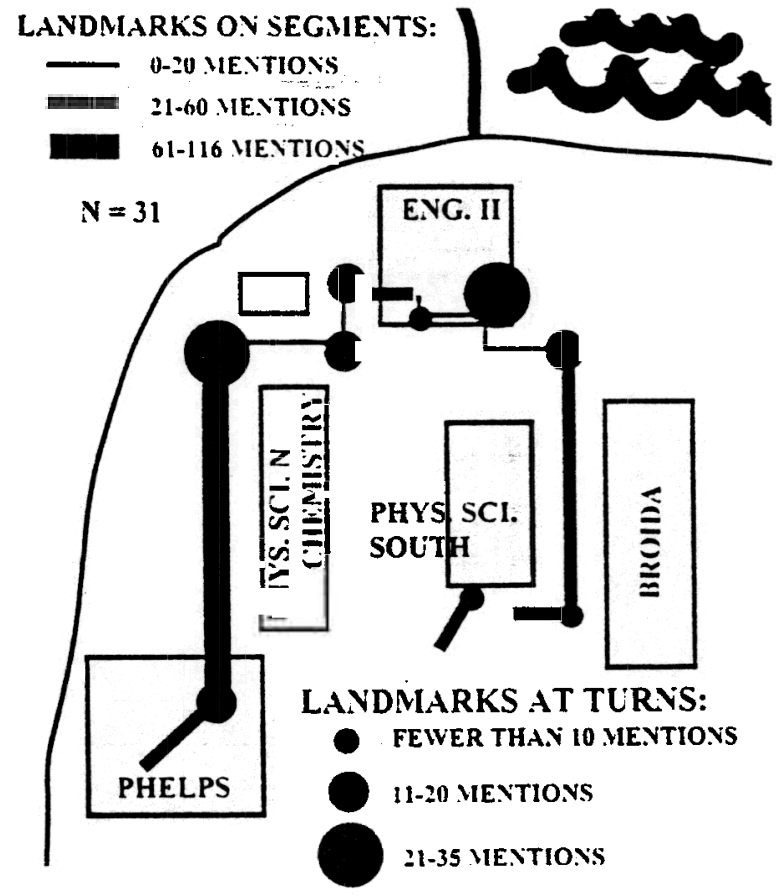

Table 1. Des Unfamiliar Rout

Route Descrif Unfamiliar

Subjective R:

Percentage of

Percentage of

Percentage of

Total Numbe

Percentage of at Cho at Pote on Ror Off Rc

Number of $E$ Number of $\mathrm{V}$

Retrace Rou

Scene Recos

Number of $\mathbf{F}$

$\underline{\text { Retrace Mea }}$ Steps

Time:

Fig. 5. Distribution of Landmarks Mentioned, Unfamiliar Route 


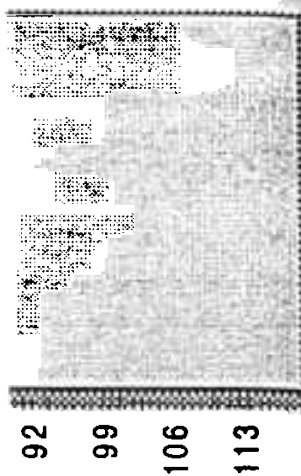

ar Route

admarks along the ong the unfamiliar ng mentioned by all 119 landmarks $\mathrm{y}$, and is relatively

$r$ route along the larks appear to be

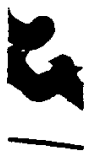

frequently mentioned on the longer segments, and they are rarely used at choice points where there are few or only one choice of which way to turn. The pattern of mentions seen here does not support the previous claim (Allen, 1997) that landmark mention increases near the end of the route, but perhaps this is due to the unfamiliarity of the terrain.

\subsection{Characteristics of Familiar and Unfamiliar Route Directions}

The elements included in both familiar and unfamiliar route directions are compared in Table 1. Subjective ratings of the two sets of route directions have similar means and standard deviations, with the more difficult route (the unfamiliar route) showing slightly more variability. Percentages are given for turns, segments, and types of landmarks for easier comparison; total numbers are used for the other measures, as no percentages can be calculated.

The percentages of turns, turns with landmarks, and segments mentioned are

Table 1. Descriptive Data: Route Direction Elements for Familiar and Unfamiliar Routes, plus Retrace and Scene Recognition Measures

Route Description Routes:

Familiar

Unfamiliar

Subjective Rating

Percentage of Turns Mentioned

Percentage of Turns w/Landmarks

Percentage of Segments Mentioned

Total Number of Landmarks

Percentage of Mentioned Landmarks

at Choice Points of Route

at Potential Choice Points

on Route, not at Choice Point

Off Route

Number of Errors

Number of Words

Mean (s.d.) Mean (s.d.)

2.9 (1.2)

$3.0(1.3)$

$64.4(27.5) \quad 44.9(23.7)$

49.7 (29.2)

$36.0(20.0)$

$75.0(20.4)$

$67.7(18.1)$

$10.0(3.2)$

$18.6(6.7)$

$29.8(10.7)$

44.1 (18.5)

$29.8(16.1)$

$2.4 \quad(3.3)$

$36.7(23.1)$

$50.5(21.5)$

$3.6 \quad(6.7)$

$3.0 \quad(4.8)$

$0.2 \quad(0.4)$

$2.6 \quad(1.9)$

$97.2(31.4)$

$185.0(77.3)$

Retrace Route (Unfamiliar Route 2)

Scene Recognition

Number of Pictures Correctly Identified

$15.4(2.0)$

Retrace Measures

Steps Off Route

$5.7(9.0)$

Times Off Route

$0.7(1.0)$

Wrong Turns

$0.4(0.6)$ 
similar for both familiar and unfamiliar routes, although the numbers are slightly higher for all measures on the familiar route. ${ }^{3}$ The percentages of the different kinds of landmarks is the most obvious difference, with an unexpectedly high percentage of on-route landmarks in the unfamiliar route, as previous research (Denis et al., 1999) has discounted their importance in favor of landmarks at choice points. Surprisingly, the correlation between the subjective ratings for the familiar and unfamiliar route directions for each participant was only 0.088 (n.s.). This low correlation of the familiar and unfamiliar route direction ratings suggests that the familiar and unfamiliar route directions may be tapping different cognitive processes.

In terms of our model (Figure 1) the two routes differ in the quality of Spatial Knowledge and in the need to make a Route Choice (for familiar only). As the retrace task suggests (see Table 3), spatial knowledge may be a limiting factor in route directions for unfamiliar routes.

\subsection{Route Direction Characteristics \& Route Direction Quality}

Correlations of the 10 route direction elements with subjective ratings of unfamiliar and familiar route directions are shown in Table 2. Six of the route direction elements had significant correlations with both the unfamiliar and familiar subjective ratings ${ }^{4}$. The unfamiliar route directions ratings were

Table 2. Correlations of Route Direction Components with Subjective Ratings

\begin{tabular}{lll} 
Component & Familiar & Unfamiliar \\
Percentage of Turns Mentioned & $.56 * *$ & $.63 * *$ \\
$\begin{array}{l}\text { Percentage of Turns with } \\
\quad \text { Landmarks }\end{array}$ & $.57 * *$ & $.56 * *$ \\
Percentage of Segments Mentioned & $.56 * *$ & $.82 * *$ \\
Total Number of Landmarks & $.49 *$ & $.70 * *$ \\
Percentage of Landmarks at: & & $.80 * *$ \\
$\quad$ Route Choice Points & .25 & .04 \\
$\quad$ Potential Choice Points & $.45 *$ & $.45 *$ \\
$\quad$ On Route, not at Choice Point & $.36 *$ & .08 \\
Off Route & .04 & .11 \\
Number of Errors & -.29 & $.61 * *$ \\
Number of Words & $.60 * *$ & \\
\hline p<.05, $* *$ p<.01 & &
\end{tabular}

${ }^{3}$ The average number of landmarks mentioned is larger for the unfamiliar route (unfamiliar $=19$, familiar $=10$ ) however the unfamiliar path is about 3 times longer.

4 Note that several significant correlations are interdependent (e.g. turn measures). significantly corre directions were $\mathbf{n}$ correlated with li directions were nc sufficient to get $\mathbf{y}$ choosing betweer specific, learned directions with on view that only lan (Denis et al., 199.

The scene rec representation of an average of 77 . three retrace mea' had quite small

During the retract once, and $29 \%$ ( 9 a wrong turn at at Table 3 sho unfamiliar and $f_{i}$ retrace measures directions for the measured by retr: model (Figure 1) by retrace ability in unfamiliar are with either set of

Table 3. Correl Scene Recognitior in

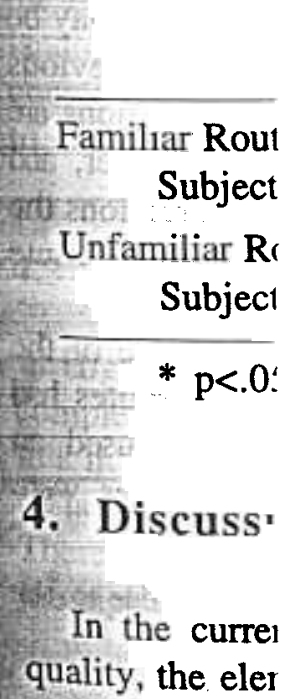
quality, the eler 
numbers are slightly ages of the different n unexpectedly high as previous research vor of landmarks at ctive ratings for the is only 0.088 (n.s.). tion ratings suggests g different cognitive

Ie quality of Spatial liliar only). As the a limiting factor in

\section{ction Quality}

bjective ratings of - Six of the route he unfamiliar and ings were

\section{sctive Ratings}

zction Rating

Unfamiliar

$.63 * *$

$.56 * *$

$.82 * *$

$.70 * *$

$.80 * *$

.04

$.45 *$

.08

.11

$.61 * *$

e unfamiliar route out 3 times longer. turn measures). significantly correlated. with landmarks at choice points, while the familiar route directions were not. The familiar route directions ratings were significantly correlated with landmarks at potential choice points, while unfamiliar route directions were not. This may reflect the role of choice point landmarks in 'being sufficient to get you there' and the role of landmarks at potential choice points in choosing between alternative routes (which is not a factor when describing a specific, learned route). The significant correlation of both kinds of route directions with on-route (not at choice point) landmarks is not consistent with the view that only: landmarks at choice points are important to quality route directions (Denis et al., 1999).

The scene recognition and retrace tasks measured ability to form a spatial representation of an unfamiliar environment. Participants correctly responded to an average of $77.1 \%$ of the scene recognition photographs (15.42 of 20). The three retrace measures (steps off the route, times off the route and wrong turns) all had quite small average errors, given the complexity of the route (Table 1). During the retrace task, $13 \%$ (4) of participants went off of the route more than once, and $29 \%$ (9) left the route one time. Only $29 \%$ (9) of the participants made a wrong turn at any of the 17 choice points (an average of 0.021 errors/turn).

Table 3 shows the correlations between the subjective ratings for the unfamiliar and familiar routes and the retrace and recognition measures. The retrace measures have a significant negative correlation with the quality of route directions for the unfamiliar route, but not the familiar route. Route memory (as measured by retrace ability) is a measure of Spatial Knowledge in the simple model (Figure 1). This supports the premise that spatial knowledge (as measured by retrace ability) is the limiting factor in the production of good route directions in unfamiliar areas. The scene recognition task was not significantly correlated with either set of route directions.

Table 3. Correlations between Subjective Route Directions Ratings and Retrace and Scene Recognition Measures

\begin{tabular}{ccc} 
& $\begin{array}{c}\text { Scene } \\
\text { Recognition }\end{array}$ & $\begin{array}{c}\text { Retrace } \\
\text { Errors }\end{array}$ \\
\hline $\begin{array}{c}\text { Familiar Route Directions } \\
\text { Subjective Rating }\end{array}$ & .15 & -.23 \\
$\begin{array}{c}\text { Unfamiliar Route Directions } \\
\text { Subjective Rating }\end{array}$ & .12 & $-.46^{*}$ \\
\hline$* \mathrm{p}<.05$ & &
\end{tabular}

\section{Discussion}

In the current study we have addressed the measurement of route direction quality, the elements of route directions and their contribution to route direction 
quality, and the differences in route directions produced about familiar and unfamiliar environments.

\subsection{Measurement of Route Direction Quality}

Our pilot study showed that reliable subjective ratings of route directions are possible. The inter-rater correlations ranged from .73 to .86. It appears that even with very minimal instructions, both informed and naive raters are using similar criteria to assign ratings to route directions for both familiar and unfamiliar routes.

\subsection{General Route Direction Characteristics}

The coding scheme for route directions in the current study improves on those used in the past (Allen, 1996; Denis, 1997; Denis et al., 1999; Vanetti and Allen, 1988) by breaking landmarks down into more categories than simply at choice points vs. not at choice points. Previous research (Denis et al., 1999) has suggested that mention of landmarks should occur only at choice points. However, the current study shows that more than $50 \%$ of the landmarks on the unfamiliar routes, and more than $40 \%$ of landmarks on familiar routes are mentioned at places other than choice points. Landmarks on route (not a choice points) are fully half of the landmarks mentioned in unfamiliar route directions, and $30 \%$ of those mentioned even in familiar environments. These on route landmarks are significantly correlated with route direction quality for both familiar and unfamiliar routes. These results are inconsistent with earlier claims (Denis et al., 1999) that only choice point landmarks are important to good route directions.

For both the familiar and unfamiliar routes, longer route directions were rated as higher quality. This result is contrary to the previous finding (Denis et al., 1999) that route directions of moderate length are rated most highly. This may be due to differences between route directions given orally (as in most previous studies) versus in written form (in the current study). When route directions are given or used orally, too much detail may pose a problem for the receiver, and more concise directions may be better. However, with written route directions the potential for overload on the receiver is less of a problem, and so longer route directions may be given. ${ }^{5}$

A second possible reason for the difference in Denis' findings and those of the current study concerns the difficulty of the route used. Denis et al.'s routes had only 2 to 4 segments, and 1 to 3 turns, whereas the current study used an unfamiliar route with 14 segments and 13 turns. ${ }^{6}$ Because Denis' routes were

\footnotetext{
5 Alternately, written directions may be more concise than oral directions, due to the ability to take as much time as necessary to plan what will be written

${ }^{6}$ Our familiar route, however, had only 4 segments and 3 turns.
} 
very simple, good descriptions likely included all of the turns and segments, and therefore extra words were likely excess detail. In this case it would make sense to say that route directions of moderate length were best. However, in the current study all of the turns and segments were not mentioned. Even for the familiar route only $64 \%$ of turns and $75 \%$ of segments were mentioned. Perhaps due to

te directions are ppears that even re using similar and uniamiliar

roves on those netti and Allen, nply at choice al., 1999) has choice points. dmarks on the iar routes are : (not a choice ute directions, hese on route r both fämiliar aims (Denis et o good route

ons were rated (Denis et al., This may be lost previous directions are receiver, and directions the longer toute

those of the 's routes had udy used an routes were 15, due to the the complexity of the routes in the current study, the longest descriptions were rated more highly because they were the most complete.

There is some consistency in the characteristics of segments and turns that are mentioned along both the familiar and unfamiliar routes (see Figure $3 a$ and $3 b$ ). For the familiar route (Figure 3a), the central two segments of the route are mentioned $100 \%$ of the time. In contrast, the shorter end segments are mentioned less often. In addition the final turn and segment may not be mentioned as frequently because the destination is visible from the end of the third segment. In general, if appears that places where there are incomplete mentions of turns or segments may be those where such information is unnecessary because of cues in the actual environment. Therefore, it may be that the location of mentioned elements is more important than the absolute number mentioned.

This characterization becomes clearer when considering the unfamiliar route (Figure 3b). Again, the longest segments are most frequently mentioned. However, in places where there are constraints on the path (e.g. walls, bushes, etc.), the turns and segments are less frequently mentioned. For the unfamiliar route, this occurs on segments 6,7 , and 11-13, and the turns within those areas (turns 6, and 11-13) all of which are very infrequently mentioned. Finally, Figure 5 provides further evidence that mentions of any route information decreases in constrained areas: in the constrained sections of the route mentioned above, there are no landmarks at all mentioned.

The landmark information given by Figure 5 is inconsistent with the claim that the mention of landmarks increases as the end of the route approaches (Allen, 1997). There is no evidence that more landmarks are mentioned close to the end of the route; in fact there are 2 segments and a choice point virtually at the end of the route where no landmarks are mentioned at all. Again, the location of the landmarks seems to be related to both availability of landmarks, and the constraints or potential choices of the specific route to be described, and possibly to the landmarks available in the spatial representation.

\subsection{Familiar versus Unfamiliar Route Directions}

Higher levels of spatial knowledge of an area should be correlated with more complete and more accurate route directions for that area. We have evidence for this in the higher percentages of turn and segment statements and larger number of landmarks per unit distance included in route directions for familiar routes. There are also significantly fewer errors in familiar route directions, attesting to the higher quality of the underlying representation in the familiar area. 
Different types of landmarks are related to quality in directions for familiar and unfamiliar routes. For both familiar and unfamiliar route directions, landmarks on route (but not at a choice point) are significantly correlated with quality of route directions and are included in the largest numbers. For only the familiar route, landmarks at potential choice points are correlated with quality and quite frequent (Table 2). For the unfamiliar route, landmarks at choice points were most highly correlated with quality and quite frequent.

This difference in landmark type use may stem from experience with the environment, and the spatial representation available when producing the route directions. In the familiar environment, it is likely that the participants had used many of the potential choice points in the past when using alternate routes. They would therefore have been aware of the landmarks at potential choice points. In contrast, the participants giving route directions for the unfamiliar environment had a relatively impoverished representation to work from. In their case, remembering the choice points actually taken, and which way to turn at these choice points was perhaps all they could remember after one exposure. It is possible that potential choice points are not represented at all in an unfamiliar environment, and do not become part of a spatial representation until much more experience is gained, or until the potential choice point is used as part of some other route.

The general model of route direction production (Figure 1) proposed that familiarity with an area is important to route direction production, since route directions are created by drawing on an underlying spatial representation. The current study supports this model: greater ability to retrace a newly learned route (a measure of spatial representation ability) is significantly correlated with higher quality route directions for a newly learned (e.g. unfamiliar) route. However, after longer exposure to an environment, the spatial representation may become rich enough that differing spatial abilities which originally created it are no longer the most important factor. This is supported by the finding that route retracing ability is not significantly correlated with the quality of route directions for a familiar setting.

\subsection{Future Directions for Research}

This research raises new questions about quality of route directions. One question is whether route directions rated as higher quality are actually more functional in getting people to their goals. That is, are the same elements of the route directions important to raters and wayfinders when assessing quality? Second, are functional measurements of quality (how well route directions facilitate the wayfinding task) reliably related to the inclusion of certain elements? This question expands the scope of the research from route direction production to include route direction comprehension issues.?

7 We are currently comparing functionality of good and poor directions for the unfamiliar route to see if the raters appear to be using functional criteria. 
for familiar and ions, landmarks with quality of lly the familiar uality and quite ice points were

ience with the ucing the route ipants had used te routes. They ice points. In ar environment In their case, $\supset$ turn at these xposure. It is I an unfamiliar til much more is part of some

proposed that on, since route ientation. The $y$ learned route ed with higher However, after y become rich : no longer the route retracing irections for a

ections. One actually more lements of the ssing quality? ute directions tain elements? production to ctions for the ia.
Third, how might the elements necessary for achieving a 'high quality' set of route directions be affected by changes in characteristics of the route itself? To determine the generalizability of the current findings, it is important to test whether the differences between the kind and frequency of elements included in good route directions for familiar versus unfamiliar routes is stable across routes of varying lengths, locations, complexity, etc. rather than idiosyncratic to the routes chosen here and in previous studies. This seems a real possibility given that none of the previous claims which were examined (e.g. density of landmarks near end of route, length of 'best' route directions, and importance of landmarks not at choice points) were supported by the current data. One suggestion is to create a number of widely varied routes using immersive or desktop virtual environments. This would provide great freedom in designing routes to test hypotheses such as the importance of constrained portions of routes to subsequent route descriptions, although there will be some loss of ecological validity. The use of both real and virtual environments can best inform a comprehensive research program on route direction quality.

\section{References}

Allen, G. L. (1997). From Knowledge to Words to Wayfinding: Issues in the Production and Comprehension of Route Directions. In S. C. Hirtle \& A. U. Frank (Eds.), Spatial information theory: A theoretical basis for GIS, pp. 363-372. Berlin: Springer-Verlag, Lecture Notes in Computer Science 1329.

Couclelis, H. (1996). Verbal directions for way-finding: Space, cognition and language. In J. Portugali (ed.) The Construction of Cognitive Maps, pp. 133-153. Dordrecht: Kluwer Academic Publishers.

Denis, M. (1997). The description of routes: A cognitive approach to the production of spatial discourse. Cahiers de Psychologie , 16, 409-458.

Denis, M., Pazzaglia, F., Cornoldi, C. \& Bertolo, L. (1999). Spatial Discourse and Navigation: An analysis of route directions in the city of Venice. Applied Cognitive Psychology, 13, 145-174.

Golding, J. M., Graesser, A. C. \& Hauselt, J. (1996). The processing of answering direction-giving questions when someone is lost on a university campus: The role of pragmatics. Applied Cognitive Psychology, 10, 23-39.

Klein, W. (1983). Deixis and Spatial Orientation in Route Directions. In H. L. Pick and L. P. Acredolo (Eds.) Spatial Orientation: Theory, research and application. New York: Plenum Press; pp. 283-312.

Kuipers, B. (1978). Modeling spatial knowledge. Cognitive Science, 2, 129-153.

Mark, D. M. (1987). On giving and receiving directions: Cartographic and cognitive issues. In Proceedings, 8th International Symposium on Computer-Assisted Cartography (pp. 562-571). Baltimore, MD.

Mark, D. M. \& Gould, M. D. Wayfinding directions as discourse: Verbal directions in Spanish and English. In J. F. Duchan, G. A. Bruder, \& L. E. Hewitt (Eds.), Deixis in Narrative: A cognitive science perspective , (pp. 387-405). Hillsdale, NJ: Lawrence Erlbaum.

Reisbeck, C. K. (1980). 'You can't miss it': Judging the clarity of directions. Cognitive Science, 4, 136-149. 
Streeter, L. A., Vitello, D., \& Wonsiewicz, S. A. (1985). How to tell people where to go: Comparing navigational aids. International Journal of Man/Machine Studies, 22, 549-562.

Talmy. L. (1983). How Language Structures Space. In H. L. Pick and L. P. Acredolo (Eds.) Spatial Orientation: Theory research and apolication. New York: Plenum Press; pp. 283-312.

Vanetti, E. J. \& Allen, G. L. (1988). Communicating environmental knowledge: The impact of verbal and spatial abilities on the production and comprehension of route directions. Environment and Behavior, 20, 667-682.

Waller, G. (1985). Linear organization of spatial instructions: Development of comprehension and production. First Language, 6, 53-67.

Wunderlich, D. \& Reinelt, R. (1982). How to get there from here. In R. Jarvella and W. Klein (Eds.), Speech. Place, and Action, pp. 183-201. Chichester, UK: Wiley.

\section{The Produ}

\section{IIntroductio}

We have severa unknown enviro the designated $\mathbf{0}$ Rodrigo, \& Zim like schemas, mi Huil, \& Umme receiver with an bas to navigate distortions in require complen are a major mod 